\title{
Systematic review and meta-analysis of isolated posterior fossa malformations on prenatal imaging (part 2): neurodevelopmental outcome
}

\author{
F. D’ANTONIO*, A. KHALIL*, C. GAREL†, G. PILU‡, G. RIZZO\$, T. LERMAN-SAGIEף, \\ A. BHIDE*, B. THILAGANATHAN*, L. MANZOLI** and A. T. PAPAGEORGHIOU* \\ "Fetal Medicine Unit, Division of Developmental Sciences, St George's University of London, London, UK; †Hôpital d'Enfants \\ Armand-Trousseau - Service de Radiologie, Cedex 12, Paris, France; $\ddagger$ Department of Obstetrics and Gynecology, Sant’Orsola-Malpighi \\ Hospital, University of Bologna, Bologna, Italy; \$Department of Obstetrics and Gynecology, Università di Roma, Tor Vergata, Rome, Italy; \\ IIFetal Neurology Clinic and Paediatric Neurology Unit, Wolfson Medical Center, Holon, Sackler School of Medicine, Tel Aviv University, \\ Tel Aviv, Israel; **Department of Medicine and Aging Sciences, University of Chieti-Pescara, and EMISAC, CeSI Biotech, Chieti, Italy
}

KEYWORDS: Blake's pouch cyst; Dandy-Walker malformation; mega cisterna magna; meta-analysis; neurodevelopmental outcome; posterior fossa anomaly; vermian hypoplasia

\begin{abstract}
Objectives Diagnosis of isolated posterior fossa anomalies in children is biased by the fact that only those that are symptomatic are brought to the attention of the appropriate clinical personnel, and the reported rate is often affected by the adoption of different nomenclature, diagnostic criteria, outcome measures, duration of follow-up and neurodevelopmental tools. The aim of this systematic review was to explore the neurodevelopmental outcome of fetuses with a prenatal diagnosis of isolated posterior fossa anomalies.
\end{abstract}

Methods MEDLINE and EMBASE were searched electronically, utilizing combinations of the relevant medical subject heading terms for 'posterior fossa' and 'outcome'. Studies assessing the neurodevelopmental outcome in children with a prenatal diagnosis of isolated posterior fossa malformations were considered eligible. The posterior fossa anomalies analyzed included Dandy-Walker malformation (DWM), mega cisterna magna (MCM), Blake's pouch cyst (BPC) and vermian hypoplasia (VH). Two authors reviewed all abstracts independently. Quality assessment of the included studies was performed using the Newcastle-Ottawa Scale for cohort studies. Meta-analyses of proportions were used to combine data, and between-study heterogeneity was explored using the $\mathrm{I}^{2}$ statistic.

Results A total of 1640 articles were identified; 95 were assessed for eligibility and a total of 16 studies were included in the systematic review. The overall rate of abnormal neurodevelopmental outcome in children with a prenatal diagnosis of DWM was $58.2 \% \quad 195 \%$ CI, $21.8-90.0 \%)$ and varied from $0-100 \%$. In those with a prenatal diagnosis of MCM, the rate of abnormal neurodevelopmental outcome was $13.8 \% \quad 195 \%$ CI, $7.3-21.9 \%)$, with a range of $0-50 \%$. There was no significant association between BPC and the occurrence of abnormal neurodevelopmental delay, with a rate of $4.7 \%(95 \%$ CI, $0.7-12.1 \%)$ and range of $0-5 \%$. Although affected by the very small number of studies, there was a non-significant occurrence of abnormal neurodevelopmental delay in children with a prenatal diagnosis of $\mathrm{VH}$, with a rate of $30.7 \% \quad 195 \% \mathrm{CI}$, $0.6-79.1 \%$ ) and range of $0-100 \%$.

Conclusions Fetuses diagnosed with isolated DWM are at high risk of abnormal neurodevelopmental outcome, while isolated MCM or BPC have a generally favorable outcome. The risk of abnormal developmental delay in cases with isolated VH needs to be further assessed. In view of the wide heterogeneity in study design, time of follow-up, neurodevelopmental tests used and the very small number of included cases, further future large prospective studies with standardized and objective protocols for diagnosis and follow-up are needed in order to ascertain the rate of abnormal neurodevelopmental outcome in children with isolated posterior fossa anomalies. Copyright (c) 2015 ISUOG. Published by John Wiley \& Sons Ltd.

Correspondence to: Dr A. T. Papageorghiou, Fetal Medicine Unit, Division of Developmental Sciences, St George's University of London, London SW17 0RE, UK (e-mail: apapageo@sgul.ac.uk)

Accepted: 16 September 2015 


\section{INTRODUCTION}

Advances in prenatal brain imaging have allowed detailed assessment of the anatomy of the posterior fossa; however, when an abnormality is found in this area of the fetal brain, parental counseling is particularly challenging because the terminology is often confusing and there are many small studies that make it difficult to reach firm conclusions regarding the long-term outcome of an individual fetus or infant. For instance, mega cisterna magna (MCM) and Blake's pouch cyst (BPC) have been reported to have a favorable outcome when isolated. Conversely, anomalies such as Dandy-Walker malformation (DWM) are commonly considered to have a poor prognosis ${ }^{1-4}$.

The lack of an objective reference standard to confirm the diagnosis after birth represents another challenge. Magnetic resonance imaging (MRI) interpretation is hampered by high rates of both false-positive and false-negative diagnoses ${ }^{5}$. Likewise, pathological confirmation of posterior fossa anomalies has a low level of concordance with prenatal imaging ${ }^{6}$. In addition, many published studies do not differentiate between cases diagnosed before and after birth. Postnatal series might be biased by the fact that only symptomatic patients come to the attention of medical practitioners, meaning that they do not reflect the natural history of the disease.

Finally, how the neurodevelopmental outcome is assessed differs between studies. This is of particular relevance because the traditional role of the cerebellum as a mere center for motor control has been reconsidered in view of recent evidence, highlighting its influence on language, socialization and cognitive functions ${ }^{7}$. Therefore, the use of different targeted neurodevelopmental tests in order to assess accurately the neurocognitive status of these patients might be necessary. The adoption of different periods of follow-up among the studies means that the rate of abnormal neurocognitive outcome remains uncertain, because some developmental anomalies may be evident only later in life, while others, labeled as abnormal early in life, are mild and may have only a small effect on the overall quality of life ${ }^{8}$.

The adoption of different nomenclature, diagnostic criteria, outcome measures, duration of follow-up and neurodevelopmental tools means that there remains significant controversy regarding neurodevelopmental outcomes in children with posterior fossa abnormalities. The aim of this systematic review was to explore the neurodevelopmental outcomes in children diagnosed in utero with isolated posterior fossa anomalies.

\section{METHODS}

\section{Protocol, eligibility criteria, information sources and search}

This review was performed according to an a-priori designed protocol and based on recommended methods for systematic reviews and meta-analyses ${ }^{9-11}$; PRISMA guidelines were followed during the conduct of this review $^{12}$.
MEDLINE and EMBASE were searched electronically on 15 February 2014, utilizing combinations of the relevant medical subject heading ( $\mathrm{MeSH}$ ) terms, keywords and word variants for 'posterior fossa', 'Dandy-Walker', 'Blake's pouch cyst', 'mega cisterna magna', 'vermian hypoplasia' or 'agenesis' and 'outcome' (Table S1). The search was then updated on 14 July 2014. The search and selection criteria were restricted to the English language. Reference lists of relevant articles and reviews were hand-searched for additional reports.

\section{Study selection, data collection and data items}

Studies were assessed according to the following criteria: population, outcome, gestational age at examination and type of imaging assessment of the posterior fossa.

Two authors (F.D., A.K.) reviewed all abstracts independently. Full-text copies of relevant papers were then obtained and relevant data regarding study characteristics and pregnancy outcome were extracted independently. Agreement regarding inclusion of studies and relevance of data was reached by consensus or by discussion with a third author (A.T.P.). If more than one study was published on the same patient cohort with identical endpoints, the report containing the most comprehensive information was included to avoid overlapping populations. For those articles in which information was not reported, but the methodology suggested that this information would have been recorded initially, the authors of the articles were contacted.

Quality assessment of the included studies was performed using the Newcastle-Ottawa Scale (NOS) for cohort studies. According to the NOS, each study was judged on three broad perspectives: selection of the study groups; comparability of the groups; and ascertainment of outcome of interest $\mathrm{t}^{13}$. Assessment of the selection of a study includes evaluation of the representativeness of the exposed cohort, selection of the non-exposed cohort, ascertainment of exposure and demonstration that the outcome of interest was not present at the start of the study. Assessment of the comparability of the study includes evaluation of the comparability of cohorts on the basis of the design or analysis. Finally, ascertainment of the outcome of interest includes evaluation of the type of assessment of the outcome of interest, and the length and adequacy of follow-up. According to NOS, a study can be awarded a maximum of one star for each numbered item within the selection and outcome categories. A maximum of two stars can be given for comparability ${ }^{13}$.

\section{Risk of bias, summary measures and synthesis of results}

The posterior fossa anomalies considered in this systematic review were defined on the basis of the morphological approach proposed by Tortori-Donati et al. ${ }^{14}$ and were: (1) DWM was defined by the classic triad of complete or partial agenesis of the cerebellar vermis, cystic dilatation of the fourth ventricle and enlarged posterior fossa with upward displacement of the tentorium, torcula and 
transverse sinuses; (2) MCM was defined as a cisterna magna measuring $>10 \mathrm{~mm}$ in the transverse cerebellar plane, and a normal cerebellar vermis; (3) BPC was defined by the presence of an upwardly displaced normal cerebellar vermis, normal-appearing fastigium, tentorium and size of the cisterna magna; (4) vermian hypoplasia (VH) was defined as a normally formed vermis but of smaller size, with the posterior fossa otherwise of normal size and anatomy.

Isolated abnormalities were defined as those posterior fossa abnormalities occurring with normal karyotype and no other associated major central nervous system (CNS) or extra-CNS anomalies detected either pre- or postnatally. In the case of DWM, ventriculomegaly was not included as an associated CNS anomaly because its development is related to dynamic changes in cerebrospinal fluid secondary to the mass effect of the cystic malformation ${ }^{14}$.

Abnormal neurodevelopmental outcome was defined as the overall presence of neurological, motor, cognitive, language or developmental deficits. A subanalysis considering the different types of neurodevelopmental abnormalities was performed whenever possible. Furthermore, the occurrence of ventriculomegaly, either before or after birth, and the need for any postnatal shunting procedure were assessed.

Only studies reporting a prenatal diagnosis of clearly defined isolated posterior fossa anomalies were considered suitable for inclusion in this systematic review. Only full-text articles were considered eligible for inclusion; case reports, conference abstracts and case series with fewer than three cases were excluded in order to avoid publication bias. In addition, we excluded from the analysis postnatal studies or studies from which cases diagnosed prenatally could not be extracted, cases of Dandy-Walker variant and those with a lack of a clear definition of the anomaly, and studies with non-isolated cases of posterior fossa anomalies. Studies published before the year 2000 were not included in the current systematic review for two related reasons: first, advances in prenatal imaging techniques are likely to have led to improvements in the diagnosis and characterization of CNS anomalies and therefore studies before this time are of little relevance to modern-day imaging; and second, older studies suffer from greater heterogeneity in definitions and nomenclature of the anomalies.

We used meta-analyses of proportions to combine data ${ }^{15,16}$. Unfortunately, the low number of studies did not permit meaningful stratified meta-analyses to explore the test performance in subgroups of patients that may be less or more susceptible to bias. Furthermore, in view of the multitude of definitions, neurodevelopmental tests used and different ages at follow-up, we also decided to provide the rate of abnormal outcome for each study individually.

Assessment of the potential publication bias was also problematic, both because of the nature of the outcome (rates with the left side limited to the value zero), which limits the reliability of funnel plots, and because of the scarce number of individual studies, which strongly limits the reliability of formal tests. Funnel plots displaying the outcome rate from individual studies vs their precision (1/standard error) were constructed with an exploratory aim. Tests for funnel-plot asymmetry were not used when the total number of publications included for each outcome was less than 10 . In this case, the power of the tests is too low to distinguish chance from real asymmetry ${ }^{17,18}$.

Between-study heterogeneity was explored using the $I^{2}$ statistic, which represents the percentage of between-study variation that is due to heterogeneity rather than chance. A value of $0 \%$ indicates no observed heterogeneity, whereas $I^{2}$ values of $\geq 50 \%$ indicate a substantial level of heterogeneity. A fixed-effects model was used if substantial statistical heterogeneity was not present. In contrast, if there was evidence of significant heterogeneity between included studies, a random-effects model was used.

All proportion meta-analyses were carried out using StatsDirect 2.7.9 (StatsDirect Ltd, Altrincham, UK) and Meta-DiSc (Meta-DiSc Statistical Methods, 2006: ftp://ftp.hrc.es/pub/programas/metadisc/Meta-DiSc StatisticalMethods.pdf).

\section{RESULTS}

\section{Study selection and characteristics}

A total of 1640 articles were identified; 95 were assessed with respect to their eligibility for inclusion (Table S2) and a total of 16 studies were included in the systematic review (Figure 1 and Table 1). These 16 studies included 158 infants with isolated posterior fossa anomalies.

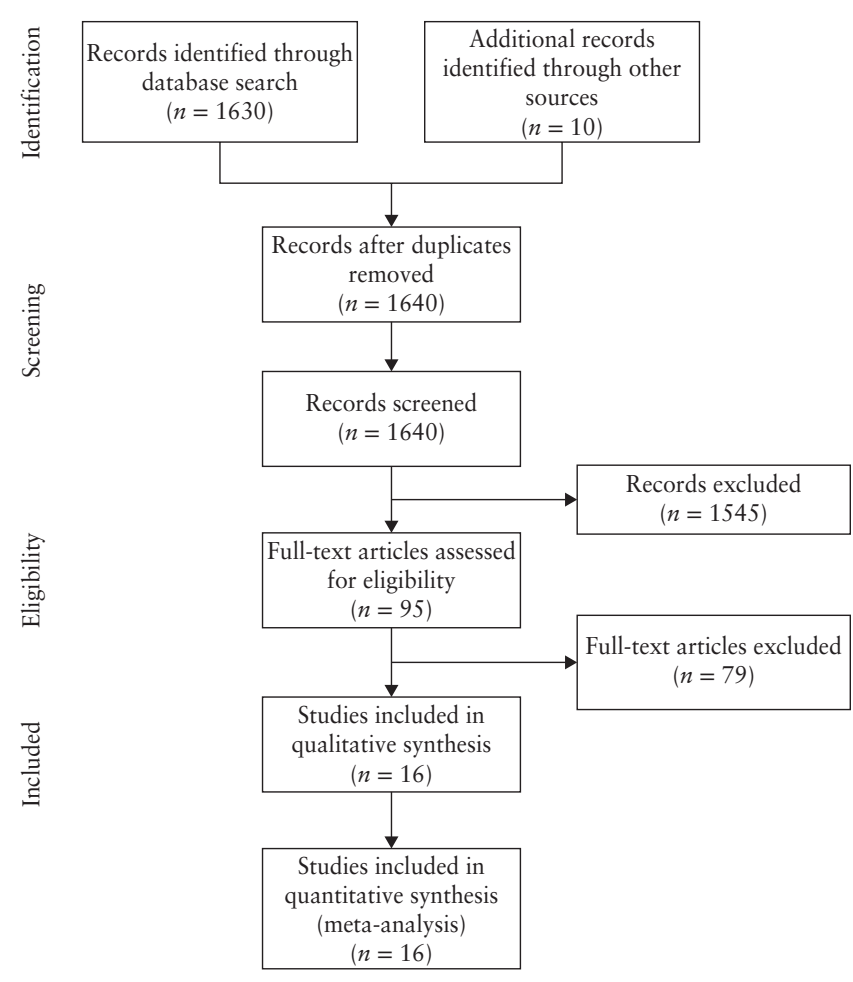

Figure 1 Flowchart summarizing selection of studies reporting on neurodevelopmental outcome of children with isolated posterior fossa anomalies diagnosed prenatally. 
Table 1 General characteristics of 16 studies reporting on neurodevelopmental outcome of children with isolated posterior fossa malformations diagnosed prenatally

\begin{tabular}{|c|c|c|c|c|c|c|}
\hline Study & Country & $\begin{array}{l}\text { Study } \\
\text { design }\end{array}$ & $\begin{array}{l}\text { Prenatal } \\
\text { imaging }\end{array}$ & $\begin{array}{l}\text { Anomalies } \\
\text { analyzed }\end{array}$ & Neurodevelopmental tool & $\begin{array}{l}\text { Age at } \\
\text { follow-up }\end{array}$ \\
\hline Tarui $(2014)^{33 *}$ & USA & Prosp. & MRI & $\mathrm{VH}$ & $\begin{array}{l}\text { Wechsler Preschool and Primary } \\
\text { Scale of Intelligence }\left(3^{\text {rd }} \text { or } 4^{\text {th }}\right. \\
\text { edn), Vineland Adaptive } \\
\text { Behaviour Scale-II, Behavior } \\
\text { Rating Inventory of Executive } \\
\text { function, Child Behavior } \\
\text { Checklist, Social Communication } \\
\text { Questionnaire }\end{array}$ & Mean 6.1 years \\
\hline Zhao $(2013)^{31}$ & China & Prosp. & US, MRI & $\mathrm{BPC}$ & Basic neurological examination & $1-3$ years \\
\hline $\begin{array}{r}\text { Vatansever } \\
(2013)^{24}\end{array}$ & UK & Prosp. & MRI & $\mathrm{MCM}$ & $\begin{array}{l}\text { Griffith Mental Developmental Scale } \\
\text { (revised), Bayley Scales of Infant } \\
\text { Development ( } 3^{\text {rd }} \text { edn) }\end{array}$ & $1-2.1$ years \\
\hline Guibaud $(2012)^{19 *}$ & France & Retro. & US, MRI & DWM & $\begin{array}{l}\text { Clinical examination and early } \\
\text { development scale }\end{array}$ & $1.9-4$ years \\
\hline $\begin{array}{l}\text { Gandolfi Colleoni } \\
(2012)^{21 *}\end{array}$ & Italy & Retro. & US, MRI & $\begin{array}{l}\text { DWM, BPC, } \\
\text { MCM, VH }\end{array}$ & Basic neurological examination & $1-5$ years \\
\hline Paladini $(2012)^{2 *}$ & Italy & Retro. & US, MRI & $\mathrm{BPC}$ & Basic neurological examination & 1 month to 3.5 years \\
\hline Patek $(2012)^{28 *}$ & USA & Retro. & US, MRI & MCM, VH & $\begin{array}{l}\text { Parental assessment, healthcare } \\
\text { assessment }\end{array}$ & 2 months to 5.5 years \\
\hline Bertucci $(2011)^{29 *}$ & Italy/Israel & Prosp. & US, MRI & BPC, MCM, VH & Basic neurological examination & Mean 2 years \\
\hline Ozkan $(2011)^{22 *}$ & Turkey & Retro. & US & DWM & Basic neurological examination & NS \\
\hline Dror $(2009)^{25 *}$ & Israel & Prosp. & US, MRI & $\mathrm{MCM}$ & $\begin{array}{l}\text { Gessell Developmental Schedules } \\
\text { and Peabody Developmental } \\
\text { Motor Scale }\end{array}$ & $16-57$ months \\
\hline Forzano $(2007)^{30 *}$ & UK & Retro. & US, MRI & $\mathrm{MCM}$ & $\begin{array}{l}\text { Semi-structured questionnaire } \\
\text { (psychomotor developmental } \\
\text { milestones, seizures) }\end{array}$ & 2 days to 3 months \\
\hline Long $(2006)^{26 *}$ & UK & Retro. & US & MCM & Basic neurological examination & 4 years \\
\hline Zalel $(2006)^{32}$ & Israel & Retro. & US, MRI & $\mathrm{BPC}$ & Basic neurological examination & $1-7.5$ years \\
\hline Has $(2004)^{20 *}$ & Turkey & Retro. & US, MRI & DWM & Basic neurological examination & $3-5.5$ years \\
\hline Leitner $(2004)^{27 *}$ & Israel & Retro. & US & MCM & Telephone interview, parental report & 3 months to 3 years \\
\hline Ecker $(2000)^{23 *}$ & USA & Retro. & US & DWM & Basic neurological examination & 6 weeks \\
\hline
\end{tabular}

Only first author of each study is given. *Additional information provided by the authors. BPC, Blake's pouch cyst; DWM, Dandy-Walker malformation; edn, edition; MCM, mega cisterna magna; MRI, magnetic resonance imaging; NS, not stated; Prosp., prospective; Retro., retrospective; US, ultrasound examination; $\mathrm{VH}$, vermian hypoplasia.

Quality assessment of the included studies was performed using NOS for cohort studies (Table 2). All studies included a relatively small number of patients and had different periods of follow-up. Furthermore, most of the included studies did not use neurodevelopmental tests for the assessment of cognitive, affective and language anomalies and motor dysfunction. Finally, in view of the different imaging protocols used and types of postnatal confirmation of the anomaly, it is possible that infants with additional anomalies were included in the study population, thus affecting the overall values of abnormal neurodevelopmental outcome reported in this systematic review.

\section{Synthesis of the results}

\section{Dandy-Walker malformation}

Five studies including 13 infants with DWM, normal karyotype and no other associated CNS or extra-CNS anomalies were included in this systematic review. All studies except one ${ }^{19}$ used a basic neurological examination to assess the neurocognitive status of the patients. The overall rate of abnormal neurodevelopmental status was $58.2 \%(95 \%$ CI, $21.8-90.0 \%)$ and varied from $0-100 \%$ (Table 3 and Figure 2a). A meta-analysis of the different neurodevelopmental abnormalities was possible only for the occurrence of abnormal motor outcome, which showed a $30.4 \%(95 \%$ CI, 8.1-59.3\%) incidence of motor delay.

The study by Guibaud et al. ${ }^{19}$ included six fetuses with isolated DWM and normal standard karyotype. After excluding three fetuses with chromosomal microdeletions, detected using high-resolution cytogenetic analysis, and one further fetus with a false-positive diagnosis, the two remaining fetuses were included in the analysis. These two cases showed normal motor outcome but exhibited mild expressive language delay, although verbal reasoning was good, on postnatal assessment. Both infants developed hydrocephaly requiring a ventriculoperitoneal shunt to decompress the raised intracranial pressure ${ }^{19}$. Has et al..$^{20}$ included three cases with a prenatal diagnosis of isolated DWM, all of which showed severe delay in motor control, although specific tests to assess extensively the cerebellar function were not performed. Two of these three infants developed hydrocephaly after birth, requiring surgery ${ }^{20}$. This finding highlights the common 
Table 2 Quality assessment of the 16 included studies according to Newcastle-Ottawa Scale

\begin{tabular}{lccc}
\hline Study & Selection & Comparability & Outcome \\
\hline Tarui $(2014)^{33}$ & $\star \star \star \star$ & $\star \star$ & $\star \star \star$ \\
Zhao $(2013)^{31}$ & $\star \star$ & $\star$ & $\star$ \\
Vatansever $(2013)^{24}$ & $\star \star \star$ & $\star$ & $\star \star$ \\
Guibaud $(2012)^{19}$ & $\star \star \star$ & $\star$ & $\star \star \star$ \\
Gandolfi Colleoni $(2012)^{21}$ & $\star \star \star$ & $\star$ & $\star \star$ \\
Paladini $(2012)^{2}$ & $\star \star \star$ & $\star$ & $\star \star$ \\
Patek $(2012)^{28}$ & $\star \star \star$ & $\star$ & $\star \star$ \\
Bertucci $(2011)^{29}$ & $\star \star \star$ & $\star$ & $\star$ \\
Ozkan $(2011)^{22}$ & $\star \star$ & $\star$ & $\star$ \\
Dror $(2009)^{25}$ & $\star \star \star$ & $\star \star$ & $\star \star \star$ \\
Forzano $(2007)^{30}$ & $\star \star$ & $\star$ & $\star \star$ \\
Long $(2006)^{26}$ & $\star \star \star$ & $\star$ & $\star$ \\
Zalel $(2006)^{32}$ & $\star \star \star$ & $\star$ & $\star \star$ \\
Has $(2004)^{20}$ & $\star \star \star$ & $\star$ & $\star \star$ \\
Leitner $(2003)^{27}$ & $\star \star$ & $\star$ & $\star$ \\
Ecker $(2000)^{22}$ & $\star \star \star$ & $\star$ & $\star$ \\
\hline Ondy & (2) & $\star$ &
\end{tabular}

Only first author of each study is given. A study can be awarded a maximum of one star for each numbered item within the selection and outcome categories. A maximum of two stars can be given for comparability.

occurrence of hydrocephaly in cases of DWM. The development of hydrocephaly is probably related to dynamic changes in the cerebrospinal fluid, secondary to the mass effect of the cystic malformation ${ }^{19}$. In the study by Gandolfi Colleoni et al. ${ }^{21}$, two children were evaluated at 2 years of age, both showing severe motor impairment, while a third child had a postnatal diagnosis of Ritscher-Schinzel syndrome, presenting with mild language and psychomotor impairment. Finally, the studies by Ozkan et al. ${ }^{22}$ and Ecker et al. ${ }^{23}$ had limited periods of follow-up and non-standardized assessment of the outcome measures (Table 4).

Overall, ventriculomegaly before or after birth occurred in $68.0 \%(95 \%$ CI, 32.3-94.5\%) of fetuses with DWM despite no associated structural anomalies and normal karyotype. Ventriculomegaly requiring a ventriculoperitoneal shunt to reduce raised intracranial pressure occurred in $62.7 \%$ (95\% CI, 27.9-91.3\%) of the cases.

\section{Mega cisterna magna}

Eight studies including 81 infants with MCM were included in the systematic review. Only two studies used specific tools to assess cerebellar function ${ }^{24,25}$. The rate of abnormal neurodevelopmental outcome was $13.8 \%(95 \%$ CI, 7.3-21.9\%) and ranged from $0-50 \%$ (Table 3 and Figure 2b). A meta-analysis of the different neurodevelopmental abnormalities was possible only for the occurrence of abnormal motor outcome, which showed an incidence of motor delay of $10.9 \%$ (95\% CI, $4.6-19.5 \%$ ).

In the largest of the studies, Dror et al..$^{25}$ included children with a prenatal diagnosis of isolated MCM with normal karyotype, evaluated by the Gesell Developmental Schedules and the Peabody Developmental Motor Scale. The age of postnatal follow-up ranged from 16 to 57 months. After excluding fetuses with additional anomalies, 17 patients were included in the analysis. Two children exhibited abnormal neurodevelopmental outcome, consisting of a generalized delay in all developmental aspects (Cases 1 and 2) and abnormal language and communication skills (Case 2) (Table 4). Children with a prenatal diagnosis of isolated MCM had significantly worse scores in their general developmental quotient, and in social interaction and visual-motor perception subtests; in contrast there was no difference in motor performance between children with a normal posterior fossa and those with MCM.

In the study by Vatansever et al. ${ }^{24}$, the authors assessed the growth trajectories of the posterior fossa using semi-automatic segmentation of reconstructed fetal brain MRI. Six fetuses with isolated MCM were included in the study and the Griffith Mental Development Scale and Bailey Scales of Infant Development were used to ascertain the neurodevelopmental outcome of these children. Half of the included cases showed some degree of neurodevelopmental delay, including visuospatial perception and attention problems. Abnormal motor development was found in 1/13 infants in the study by Long et al. ${ }^{26}$ and in 3/9 in that by Leitner et al. ${ }^{27}$. Neither study used specific tests to assess cerebellar function, and in the study by Leitner et al., the neurodevelopmental status was assessed by telephone interview conducted by pediatric neurologists ${ }^{27}$. In the study by Gandolfi Colleoni et al. ${ }^{21}$, 16 cases with isolated MCM were analyzed and two children were found to have mild language disorder at around 3 years of age.

All other studies did not report any significant neurological anomaly in children with a prenatal diagnosis of isolated MCM, although no specific neurodevelopmental tool was used (Table 4) ${ }^{28-30}$.

Overall, ventriculomegaly before or after birth occurred in $2.3 \%(95 \% \mathrm{CI}, 0.1-12.3 \%)$ of cases of MCM

Table 3 Pooled proportions (PP) for occurrence of abnormal neurodevelopmental outcome in infants with prenatal diagnosis of posterior fossa anomaly

\begin{tabular}{|c|c|c|c|c|c|}
\hline Anomaly & Studies (n) & Fetuses (n/N) & $\operatorname{Raw}(95 \%$ CI) (\%) & $\mathrm{I}^{2}(\%)$ & PP $(95 \%$ CI) (\%) \\
\hline DWM & 5 & $8 / 13$ & $61.54(31.6-86.1)$ & 54.2 & $58.15(21.8-90.0)$ \\
\hline MCM & 8 & $11 / 81$ & $13.58(7.0-23.0)$ & 36.2 & $13.8(7.3-21.9)$ \\
\hline BPC & 5 & $1 / 46$ & $2.17(0.1-11.5)$ & 0.0 & $4.7(0.7-12.1)$ \\
\hline $\mathrm{VH}$ & 4 & $3 / 18$ & $16.67(3.6-41.4)$ & 77.7 & $30.7(0.6-79.1)$ \\
\hline
\end{tabular}

BPC, Blake's pouch cyst; DWM, Dandy-Walker malformation; MCM, mega cisterna magna; VH, vermian hypoplasia. 

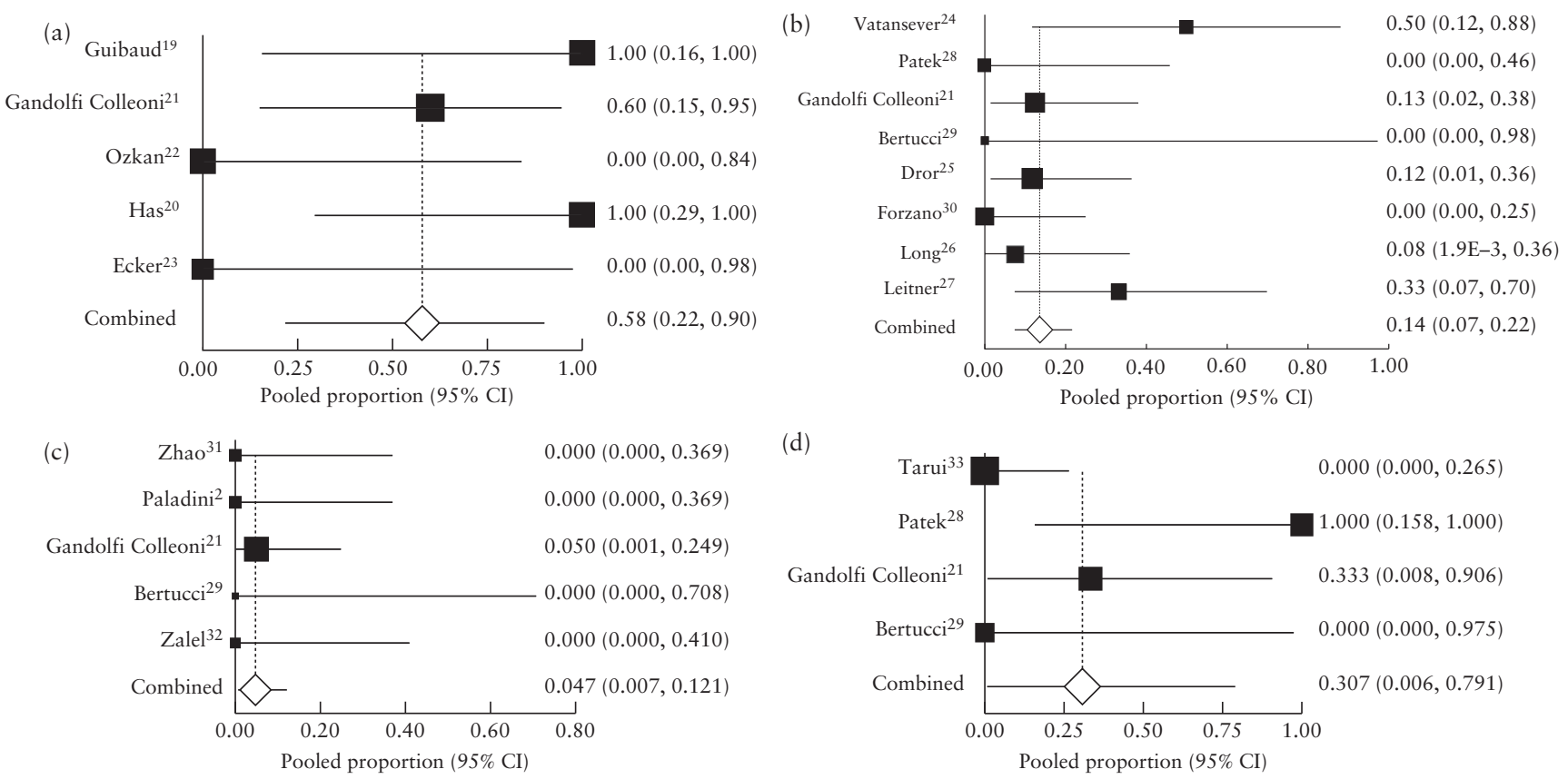

Figure 2 Pooled proportions of occurrence of abnormal overall neurodevelopmental outcome in infants with prenatal diagnosis of isolated posterior fossa malformations: (a) Dandy-Walker malformation; (b) mega cisterna magna; (c) Blake's pouch cyst; (d) vermian hypoplasia. Only first author of each study is given.

with no associated structural anomalies and normal karyotype, but in no case included in this review was a ventriculoperitoneal shunt needed (pooled proportion (PP), 0\% (95\% CI, 0-8.2\%)).

\section{Blake's pouch cyst}

Five studies including 46 infants with a prenatal diagnosis of isolated BPC were included in this review. No study used a specific neurodevelopmental test to assess cerebellar function. The age of follow-up varied from 1 month to 10 years. There was no significant association between BPC and the occurrence of abnormal neurodevelopmental delay (PP, 4.7\% (95\% CI, 0.7-12.1\%); range, 0-5\%; Table 3 and Figure 2c). No fetus tested for motor control showed an abnormal outcome (PP, 0\% (95\% CI, $0-13.2 \%))$.

In the study by Gandolfi Colleoni et al. ${ }^{21}$, the authors included 20 infants with a prenatal diagnosis of BPC, of which only one showed mild psychomotor disorder at 3 years. In the other included studies, no case of abnormal neurodevelopmental outcome was found ${ }^{2,29,31,32}$, although no specific neurodevelopmental tool was used (Table 4).

The rate of ventriculomegaly occurring either before or after birth was $12.4 \%$ (95\% CI, 2.9-27.1\%) but it did not require shunting in any of the cases (PP, 0\% (95\% CI, $0-15.4 \%))$.

\section{Vermian hypoplasia}

Four studies including 18 infants with a prenatal diagnosis of $\mathrm{VH}$ were included in this review. The duration of follow-up ranged from 6 months to 10 years.
There was high heterogeneity among the included studies, which reported a non-significant occurrence of abnormal neurodevelopmental delay among these children (PP, 30.7\% (95\% CI, 0.6-79.1\%); range, 0-33\%; Table 3 and Figure 2d). Of the included fetuses, none had abnormal motor outcome at assessment, performed at a variety of ages (PP, $0 \%(95 \% \mathrm{CI}, 0-18.5 \%)$ ).

In the largest series in this review, Tarui et al. ${ }^{33}$ prospectively followed 20 children with a prenatal diagnosis of $\mathrm{VH}$ on MRI, with targeted neurodevelopmental tests including the assessment of cognitive, affective, language and behavioral measures at school age (Table 4). When considering only cases with isolated $\mathrm{VH}$ and a confirmed postnatal diagnosis, all 12 children had normal neurodevelopmental outcome.

No fetus with $\mathrm{VH}$ included in this review required a ventriculoperitoneal shunt (PP, 0\% $\quad(95 \% \quad \mathrm{CI}$, $0-24.7 \%))$.

\section{DISCUSSION}

\section{Summary of evidence}

The findings of this systematic review show that children with a prenatal diagnosis of isolated DWM are at increased risk of abnormal neurodevelopmental outcome. Isolated MCM has a generally good outcome, although a small proportion of children may exhibit variable degrees of developmental delay. Isolated BPC is a benign condition and the rate of abnormal neurodevelopmental delay seems to be low. In view of the very small number of included studies, no clear evidence can be extracted for isolated $\mathrm{VH}$. 

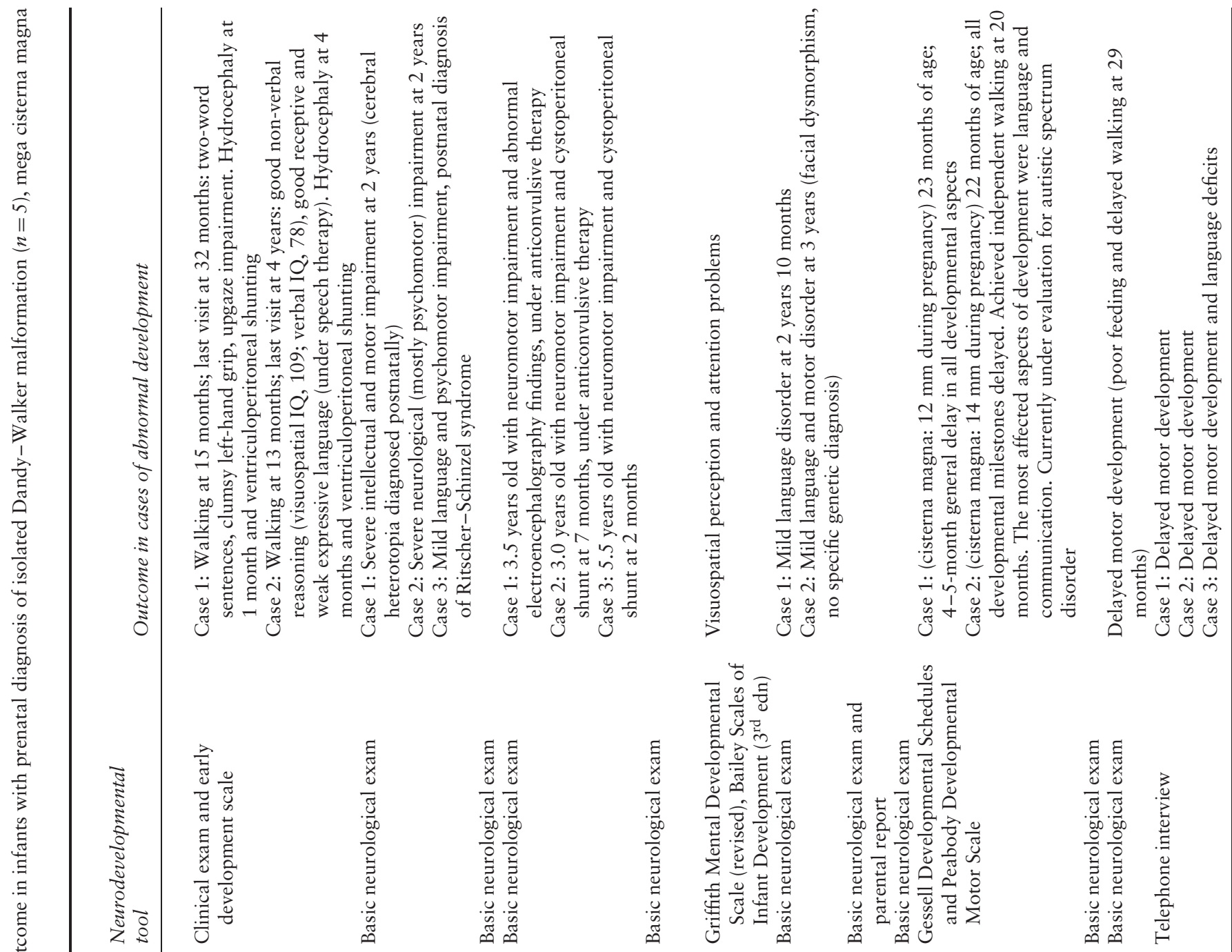

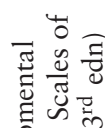
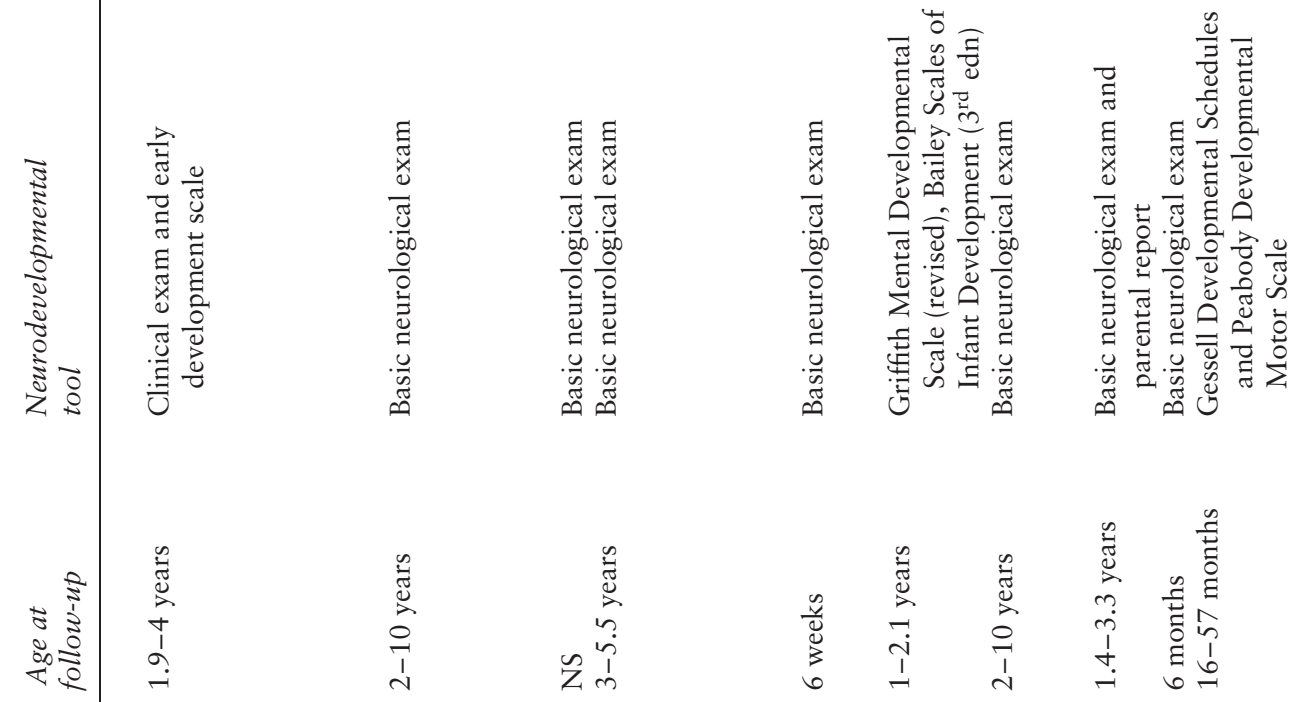

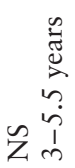
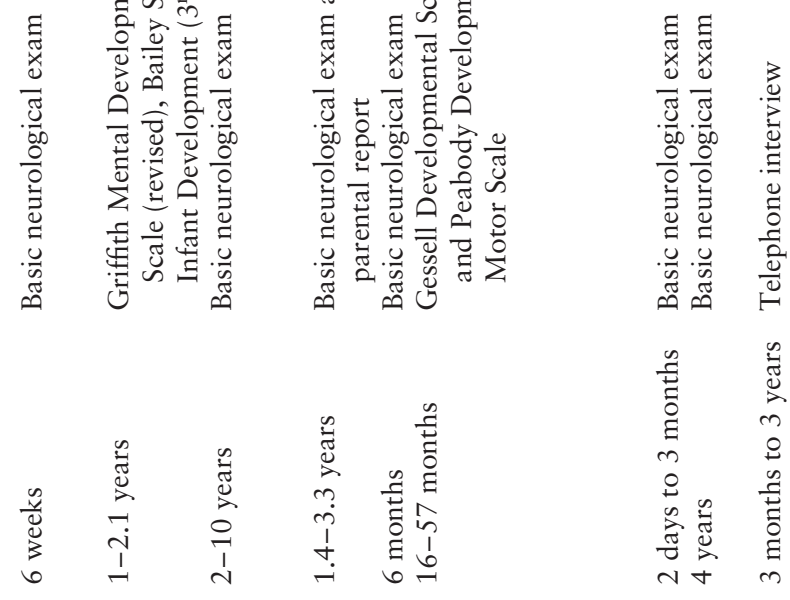

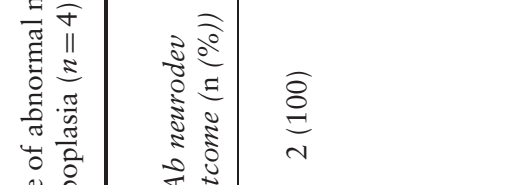

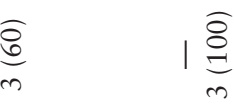

$1 \quad \frac{0}{2}$

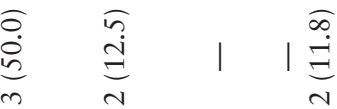

$\mid \stackrel{\substack{n \\=}}{\stackrel{n}{n}}$

莺主

跣.

言

些要

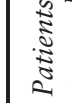

离

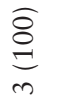

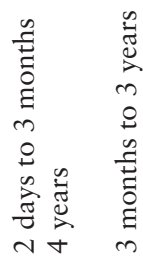

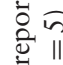

要这

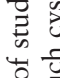

政

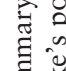

端

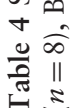

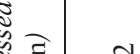

$\operatorname{rm}$

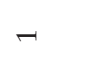

$\stackrel{-10}{2}$

$\stackrel{2}{\rightarrow} a$

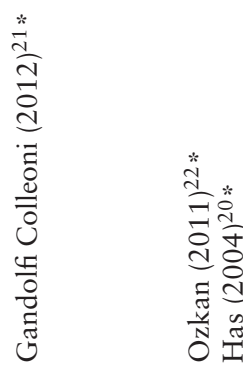

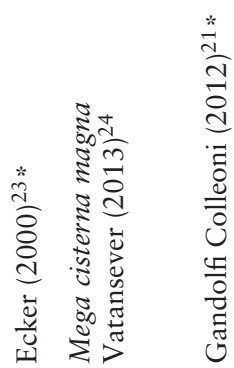
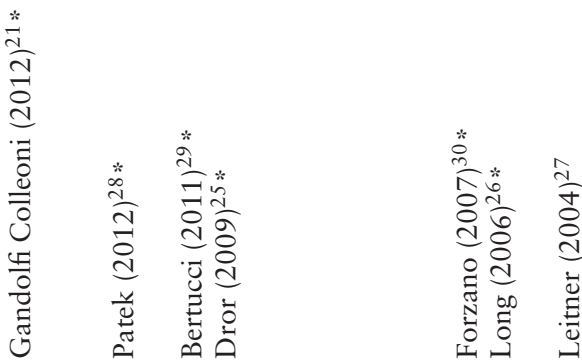


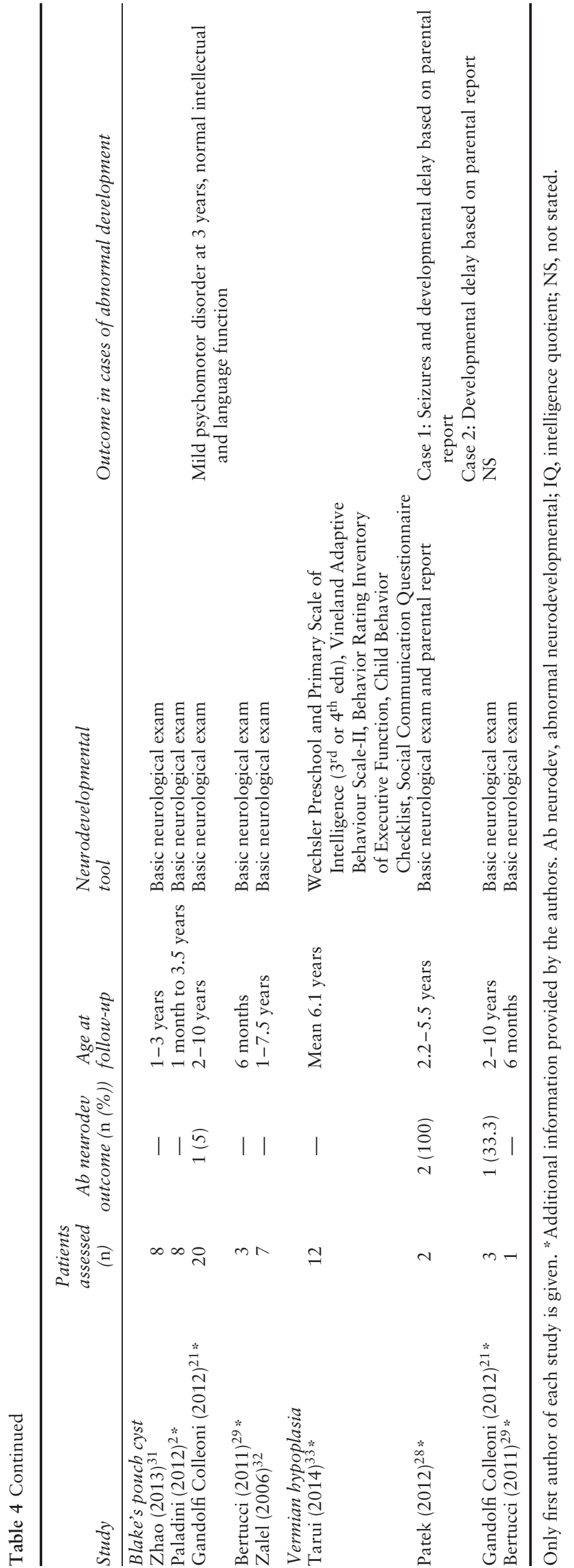

\section{Limitations of the study}

The small sample size of the included studies, high degree of variability in the definition of the different posterior fossa anomalies and differences in age at follow-up represent the major limitations of this review. A basic neurological examination, as carried out in most of the published studies, may not be sufficient to determine the neurodevelopmental status of these children and more accurate tests investigating cognitive, affective and behavioral functions are needed in order to ascertain the actual rate of abnormal development. Furthermore, cases labeled as isolated may have had subtle undiagnosed associated chromosomal or structural anomalies ${ }^{34}$. Postnatal confirmation of posterior fossa anomalies can also be challenging, with high rates of false-positive diagnoses reported in the literature ${ }^{5}$. The lack of a standardized protocol for postnatal assessment in most of the included studies did not enable a precise estimation of the exact number of diagnoses confirmed after birth. Moreover, confirmation of the diagnosis using postnatal imaging was not performed in some of the included cases. It is therefore plausible that limitations in study design, sample size, data extraction and outcomes observed might bias the findings of the current review.

\section{Implications for clinical practice}

Prenatal counseling when a fetus is diagnosed with a posterior fossa anomaly is challenging. Correctly defining posterior fossa anomalies is the first step in an optimal diagnostic approach ${ }^{35,36}$; multiplanar assessment of the posterior fossa using axial, sagittal and coronal planes is necessary in order to define precisely these conditions, especially because anomalies with a similar appearance in the axial plane may have different appearances in other planes, and may be associated with different outcomes.

The factors already outlined, such as length of follow-up, neurodevelopmental tool adopted, age at assessment, presence of additional anomalies and choice of an appropriate control group are all relevant. The term 'neurodevelopmental outcome' can also be misleading and inappropriate when considering brain anomalies because it encompasses a wide spectrum of signs with different underlying disorders and pathological processes, which are not always easily measured and which represent a continuous interaction between pathological, environmental and adaptive factors.

The study by Klein et al. ${ }^{3}$ highlights the need for new research aimed at finding reliable prognostic imaging markers; the authors reviewed retrospectively the charts of 26 patients with a diagnosis of DWM. They found that no patient in the group with normal vermis morphology had associated brain malformations and the majority had normal outcome, while all those with a dysplastic vermis had associated brain anomalies and abnormal outcome. As all patients in the second group had associated brain anomalies, it was not possible to assess whether the presence of the normal vermian anatomy was associated independently with a better outcome ${ }^{3}$. 
The feasibility of detailed evaluation of vermian anatomy during prenatal life and its independent role in predicting neurodevelopmental outcome have yet to be established ${ }^{19}$.

Isolated MCM is a relatively common finding. The rate of chromosomal abnormalities and additional CNS and extra-CNS structural anomalies that are not detected on ultrasound is $\operatorname{low}^{37}$. In the current review, most of the included studies reported a normal or borderline neurodevelopmental outcome in the majority of children with isolated MCM. The pathophysiology of isolated MCM has not been elucidated completely yet and it is not clear whether expansion of the posterior fossa by fluid is a pathological development or represents a normal variant. Dror et al. ${ }^{25}$ suggested that children with isolated MCM had lower developmental, visual motor and social performance than did controls. However, all the mean values for the neurodevelopmental measures observed were within normal range, suggesting a generally favorable outcome.

Failure of fenestration of the posterior membranous area leads to the persistence of Blake's pouch ${ }^{38}$. On imaging, BPC is characterized by the presence of an upward displacement of a normal cerebellar vermis, normal fastigium, tentorium and size of the cisterna magna. Tetraventricular hydrocephaly is an associated finding commonly reported postnatally. Although none of the included studies used specific tools to assess cerebellar function, the findings of this review suggest a generally favorable outcome.

Data on the prognosis of children with isolated $\mathrm{VH}$ in this review are debatable. In view of the very small number of cases included, no robust evidence can be confidently extrapolated. The results from this meta-analysis are surprising and disagree with what is observed after birth, where $\mathrm{VH}$, even if isolated, has been reported to be associated with developmental delay. We might speculate that the main bias is due to the definition of $\mathrm{VH}$ before birth - many cases labeled as hypoplasia during the prenatal period may actually correspond to a normal vermis, theoretically explaining the reason behind the favorable outcome reported in this meta-analysis. In the collective authors' experience, prenatal diagnosis of $\mathrm{VH}$ is affected by high rates of false-positive cases, with most of the cases found to be BPC at birth ${ }^{5}$.

\section{Implications for research}

The wide heterogeneity in diagnostic criteria, nomenclature and outcome definitions highlights the urgent need for prospective studies that standardize objectively the classification and prognosis of these anomalies. Future research should aim at describing objectively the different posterior fossa anomalies and correlating them with robust long-term neurodevelopmental measures.

\section{Conclusions}

Isolated DWM is associated with an increased risk of abnormal neurodevelopmental outcome, while isolated
MCM and BPC have a generally favorable outcome. In view of the very small number of patients tested and lack of an objective prenatal definition, the risk of abnormal developmental delay in cases with isolated $\mathrm{VH}$ needs to be further assessed. Future large prospective studies with standardized and objective protocols for diagnosis and follow-up are needed in order to ascertain the rate of abnormal neurodevelopmental outcome in children with isolated posterior fossa anomalies.

\section{ACKNOWLEDGMENTS}

We thank Prof. L. Guibaud, Prof. D. Paladini, Prof. Y. Zalel, Prof. T. Tarui, Prof. B. Benacerraf, Prof. R. Palma-Dias, Prof. R. Has, Dr E. Bertucci, Dr K. Reidy, Dr R. Ghali, Dr J. Garcia-Flores, Dr A. Borrell, Dr Y. Leitner, Dr G. Tonni, Dr A. Long, Dr K. Patek, Dr C. Spaeth, Dr M. Scheier, Dr R. Lachmann, Dr J.L. Ecker, Dr N. Kölble, Dr T. Harper, Dr P.H. Tang, Dr Z. Ozkan and Dr R. Russo for their contribution to this systematic review in terms of support and additional data supplied.

\section{REFERENCES}

1. Bolduc ME, Limperopoulos C. Neurodevelopmental outcomes in children with cerebellar malformations: a systematic review. Dev Med Child Neurol 2009; 51 $256-267$.

2. Paladini D, Quarantelli M, Pastore G, Sorrentino M, Sglavo G, Nappi C. Abnormal or delayed development of the posterior membranous area of the CNS: anatomy, ultrasound diagnosis, natural history and outcome of Blake's pouch cyst in the fetus. Ultrasound Obstet Gynecol 2012; 39: 279-287.

3. Klein O, Pierre-Kahn A, Boddaert N, Parisot D, Brunelle F. Dandy-Walker malformation: prenatal diagnosis and prognosis. Childs Nerv Syst 2003; 19: 484-489.

4. Guibaud L, Larroque A, Ville D, Sanlaville D, Till M, Gaucherand P, Pracros JP, des Portes V. Prenatal diagnosis of 'isolated' Dandy-Walker malformation: imaging findings and prenatal counselling. Prenat Diagn 2012; 32: 185-193.

5. Limperopoulos C, Robertson RL Jr, Khwaja OS, Robson CD, Estroff JA, Barnewolt C, Levine D, Morash D, Nemes L, Zaccagnini L, du Plessis AJ. How accurately does current fetal imaging identify posterior fossa anomalies? AJR Am J Roentgenol 2008; 190: $1637-1643$.

6. Carroll SG, Porter H, Abdel-Fattah S, Kyle PM, Soothill PW. Correlation of prenatal ultrasound diagnosis and pathologic findings in fetal CNS abnormalities. Ultrasound Obstet Gynecol 2000; 16: 149-153.

7. Robinson AJ. Inferior vermian hypoplasia - preconception, misconception. Ultrasound Obstet Gynecol 2014; 43: 123-136.

8. Marlow N. Measuring neurodevelopmental outcome in neonatal trials: a continuing and increasing challenge. Arch Dis Child Fetal Neonatal Ed 2013; 98: F554-F558.

9. Henderson LK, Craig JC, Willis NS, Tovey D, Webster AC. How to write a Cochrane systematic review. Nephrology (Carlton) 2010; 15: 617-624.

10. NHS Centre for Reviews and Dissemination. Systematic Reviews: CRD's Guidance for Undertaking Reviews in Healthcare. University of York: York, UK, 2009

11. Leeflang MM, Deeks JJ, Gatsonis C, Bossuyt PM. Cochrane Diagnostic Test Accuracy Working Group. Systematic reviews of diagnostic test accuracy. Ann Intern Med 2008; 149: 889-897.

12. PRISMA statement. http://www.prisma-statement.org/

13. Wells GA, Shea B, O'Connell D, Peterson J, Welch V, Losos M, Tugwell P. [Newcastle-Ottawa Scale for assessing the quality of nonrandomised studies in meta-analyses.] http://www.ohri.ca/programs/clinical_epidemiology/oxford.asp

14. Tortori-Donati P, Rossi A, Biancheri R. Brain malformations. In Pediatric Neuroradiology, Tortori-Donati P (ed). Springer: New York, 2005; 71-198.

15. Manzoli L, De Vito C, Salanti G, D’Addario M, Villari P, Ioannidis JP. Meta-analysis of the immunogenicity and tolerability of pandemic influenza A 2009 (H1N1) vaccines. PLOS One 2011; 6: e24384.

16. Hunter JP, Saratzis A, Sutton AJ, Boucher RH, Sayers RD, Bown MJ. In meta-analyses of proportion studies, funnel plots were found to be an inaccurate method of assessing publication bias. J Clin Epidemiol 2014; 67: 897-903.

17. Egger M, Davey Smith G, Schneider M, Minder C. Bias in meta-analysis detected by a simple, graphical test. BMJ 1997; 315: 629-634.

18. Higgins JPT, Green S (eds). Cochrane Handbook for Systematic Reviews of Interventions Version 5.0.2 [updated September 2009]. The Cochrane Collaboration, 2009. Available from www.cochrane-handbook.org

19. Guibaud L, Larroque A, Ville D, Sanlaville D, Till M, Gaucherand P, Pracros JP, des Portes V. Prenatal diagnosis of 'isolated' Dandy-Walker malformation: imaging findings and prenatal counselling. Prenat Diagn 2012; 32: 185-193. 
20. Has R, Ermiş H, Yüksel A, Ibrahimoğlu L, Yildirim A, Sezer HD, Başaran S. Dandy-Walker malformation: a review of 78 cases diagnosed by prenatal sonography. Fetal Diagn Ther 2004; 19: 342-347.

21. Gandolfi Colleoni G, Contro E, Carletti A, Ghi T, Campobasso G, Rembouskos G, Volpe G, Pilu G, Volpe P. Prenatal diagnosis and outcome of fetal posterior fossa fluid collections. Ultrasound Obstet Gynecol 2012; 39: 625-631.

22. Ozkan ZS, Gilgin H, Aygün HB, Deveci D, Simşek M, Kumru S, Yüce H. Our clinical experience about prenatal diagnosis and neonatal outcomes of fetal central nervous system anomalies. J Matern Fetal Neonatal Med 2011; 24: 502-505.

23. Ecker JL, Shipp TD, Bromley B, Benacerraf B. The sonographic diagnosis of Dandy-Walker and Dandy-Walker variant: associated findings and outcomes. Prenat Diagn 2000; 20: 328-332.

24. Vatansever D, Kyriakopoulou V, Allsop JM, Fox M, Chew A, Hajnal JV, Rutherford MA. Multidimensional analysis of fetal posterior fossa in health and disease. Cerebellum 2013; 12: 632-644.

25. Dror R, Malinger G, Ben-Sira L, Lev D, Pick CG, Lerman-Sagie T. Developmental outcome of children with enlargement of the cisterna magna identified in utero. $J$ Child Neurol 2009; 24: 1486-1492.

26. Long A, Moran P, Robson S. Outcome of fetal cerebral posterior fossa anomalies. Prenat Diagn 2006; 26: 707-710.

27. Leitner Y, Goez H, Gull I, Mesterman R, Weiner E, Jaffa A, Harel S. Antenatal diagnosis of central nervous system anomalies: can we predict prognosis? J Child Neurol 2004; 19: 435-438.

28. Patek KJ, Kline-Fath BM, Hopkin RJ, Pilipenko VV, Crombleholme TM, Spaeth CG. Posterior fossa anomalies diagnosed with fetal MRI: associated anomalies and neurodevelopmental outcomes. Prenat Diagn 2012; 32: 75-82.

29. Bertucci E, Gindes L, Mazza V, Re C, Lerner-Geva L, Achiron R. Vermian biometric parameters in the normal and abnormal fetal posterior fossa: three-dimensional sonographic study. J Ultrasound Med 2011; 30: 1403-1410.
30. Forzano F, Mansour S, Ierullo A, Homfray T, Thilaganathan B. Posterior fossa malformation in fetuses: a report of 56 further cases and a review of the literature. Prenat Diagn 2007; 27: 495-501.

31. Zhao D, Liu W, Cai A, Li J, Chen L, Wang B. Quantitative evaluation of the fetal cerebellar vermis using the median view on three-dimensional ultrasound. Prenat Diagn 2013; 33: 153-157.

32. Zalel Y, Gilboa Y, Gabis L, Ben-Sira L, Hoffman C, Wiener Y, Achiron R. Rotation of the vermis as a cause of enlarged cisterna magna on prenatal imaging. Ultrasound Obstet Gynecol 2006; 27: 490-493.

33. Tarui T, Limperopoulos C, Sullivan NR, Robertson RL, du Plessis AJ. Long-term developmental outcome of children with a fetal diagnosis of isolated inferior vermian hypoplasia. Arch Dis Child Fetal Neonatal Ed 2014; 99: F54-F58.

34. Shaffer LG, Rosenfeld JA, Dabell MP, Coppinger J, Bandholz AM, Ellison JW, Ravnan JB, Torchia BS, Ballif BC, Fisher AJ. Detection rates of clinically significant genomic alterations by microarray analysis for specific anomalies detected by ultrasound. Prenat Diagn 2012; 32: 986-995.

35. Guibaud L, des Portes V. Plea for an anatomical approach to abnormalities of the posterior fossa in prenatal diagnosis. Ultrasound Obstet Gynecol 2006; 27: $477-481$.

36. Garel C. Posterior fossa malformations: main features and limits in prenatal diagnosis. Pediatr Radiol 2010; 40: 1038-1045.

37. D'Antonio F, Khalil A, Garel C, Pilu G, Rizzo G, Lerman-Sagie T, Bhide A, Thilaganathan B, Manzoli L, Papageorghiou AT. Systematic review and meta-analysis of isolated posterior fossa malformations on prenatal ultrasound imaging (part 1): nomenclature, diagnostic accuracy and associated anomalies. Ultrasound Obstet Gynecol 2016; 47: 690-697.

38. Shekdar K. Posterior fossa malformations. Semin Ultrasound CT MR 2011; 32 $228-241$.

\section{SUPPORTING INFORMATION ON THE INTERNET}

The following supporting information may be found in the online version of this article:

Table S1 Search strategy - PRISMA 2009 checklist

Table S2 Excluded studies and reason for exclusion 


\section{RESUMEN}

Objetivos El diagnóstico de anomalías aisladas de la fosa posterior en niños/as está sesgado por el hecho de que sólo se notifica al personal clínico apropiado cuando presentan síntomas, y la tasa reportada se ve a menudo afectada por la adopción de nomenclatura diferente, los criterios de diagnóstico, las medidas del resultado, la duración del seguimiento y las herramientas de desarrollo neurológico. El objetivo de esta revisión sistemática fue explorar el resultado del desarrollo neurológico de los fetos con diagnóstico prenatal de anomalías aisladas de la fosa posterior.

Métodos Se hicieron búsquedas electrónicas en MEDLINE y EMBASE, utilizando combinaciones de los términos médicos más relevantes para 'fosa posterior' y 'resultado'. Se consideraron adecuados los estudios que evaluaron el resultado del desarrollo neurológico en niños/as con un diagnóstico prenatal de malformaciones aisladas de la fosa posterior. Entre las anomalías de la fosa posterior analizadas están el síndrome de Dandy-Walker (SDW), la megacisterna magna (MCM), el quiste de la Bolsa de Blake (BPC, por sus siglas en inglés) y la hipoplasia vermiana (VH, por sus siglas en inglés). Todos los resúmenes fueron revisados de forma independiente por dos de los autores. La evaluación de calidad de los estudios incluidos se realizó mediante la escala Newcastle-Ottawa para estudios de cohortes. Se utilizaron metaanálisis de proporciones para la combinación de datos, y la heterogeneidad entre estudios se examinó mediante el estadístico $\mathrm{I}^{2}$.

Resultados Se identificaron un total de 1640 artículos, de los cuales se evaluó la elegibilidad de 95 y se incluyó un total de 16 estudios en la revisión sistemática. La tasa global de resultado de desarrollo neurológico anormal en niños/as con un diagnóstico prenatal de SDW fue del 58,2\% (IC del 95\%, 21,8-90,0\%), con un rango de $0-100 \%$. En niños/as con un diagnóstico prenatal de MCM, la tasa de resultado de desarrollo neurológico anormal fue del 13,8\% (IC del 95\%, $7,3-21,9 \%)$, con un rango de $0-50 \%$. No se encontró una asociación significativa entre el BPC y la presencia de retraso de desarrollo neurológico anormal, con una tasa del $4,7 \%$ (IC del $95 \%, 0,7-12,1 \%$ ) y un rango de $0-5 \%$.Se encontró un resultado no significativo de casos con retraso del desarrollo neurológico anormal en niños/as con un diagnóstico prenatal de $\mathrm{VH}$, con una tasa del $30,7 \%$ (IC del $95 \%, 0,6-79,1 \%$ ) y un rango de $0-100 \%$, Aunque afectado por el escaso número de estudios

Conclusiones Los fetos diagnosticados con SWD aislado poseen un alto riesgo de resultado de desarrollo neurológico anormal, mientras que el MCM o BPC aislados tienen, en general, un resultado favorable. El riesgo de retraso en el desarrollo anormal en casos con VH aislada debe estudiarse más todavía. En vista de la gran heterogeneidad en el diseño de los estudios, el tiempo de seguimiento, las pruebas de desarrollo neurológico empleadas y el pequeño número de casos incluidos, será necesario realizar en el futuro estudios prospectivos más amplios con protocolos objetivos y estandarizados para el diagnóstico y el seguimiento, con el fin de determinar la tasa de resultados de desarrollo neurológico anormal en niños/as con anomalías aisladas de la fosa posterior.

目的: 由于仅有症状的患儿才会引起相应临床医务人员的注意, 因此儿童中单发后桢窝异常的诊断存在偏倚, 并且研究报道的发病率 常常由于采用不同的命名、诊断标准、结局测量、随访时间以及神经发育检查方法而受到影响。本篇系统综述的目的是探讨产前诊断 为单发后颅窝畸形胎儿的神经发育结局。

方法: 计算机检索 MEDLINE 和 EMBASE 数据库, 组配检索相关医学主题词 “后颓窝” 和 “结局”。对产前诊断为单发后颓窝畸形的 儿童神经发育结局进行评估的研究符合入选标准。分析的后颖窝异常包括 Dandy-Walker 畸形 (Dandy-Walker malformation, DWM）、巨大枕大池（mega cisterna magna, MCM）、Blake 霊肿（Blake's pouch cyst, BPC) 和小脑蚂部发育不全（vermian hypoplasia, VH）。由 2 位作者独立汶览所有摘要。采用队列研究 Newcastle-Ottawa 量表对纳入研究的质量进行评估。对比例指标进 行荟萃分析来合并数据, 采用 $I^{2}$ 统计量探讨研究间的异质性。

结果: 共检索到 1640 篇文献, 其中 95 篇符合入选标准, 系统综述共纳入 16 项研究。产前诊断为 DWM 的儿童中异常神经发育结局的 总发生率为 $58.2 \%(95 \% \mathrm{CI}, 21.8 \% \sim 90.0 \%)$ ，范围为 $0 \sim 100 \%$ 。产前诊断为 $\mathrm{MCM}$ 的儿童中，异常神经发育结局发生率为 $13.8 \%$ ( $95 \%$ CI, $7.3 \% ~ 21.9 \%)$, 范围为 $0250 \%$ 。 BPC 与异常神经发育迟缓的发生无明显相关性, 发生率为 $4.7 \%(95 \% \mathrm{CI}$, $0.7 \% \sim 12.1 \%)$, 范围为 $0 \sim 5 \%$ 。尽管研究数量极少, 但产前诊断为 $\mathrm{VH}$ 的儿童中异常神经发育迟缓的发生无明显差异, 发生率为 $30.7 \%(95 \%$ CI， $0.6 \% \sim 79.1 \%)$ ，范围为 $0 \sim 100 \%$ 。

结论: 诊断为单发 DWM 的胎儿出现异常神经发育结局的风险极高, 单发 MCM 或 BPC 通常结局较好。应进一步评估单发 VH 患儿中 异常发育迟缓的风险。考虑到研究设计存在很大异质性、随访的时间、所用的神经发育检查以及纳入病例数极少, 为了确定单发后颖 窝异常的儿童中异常神经发育结局的发生率, 需要应用标准、客观的诊断和随访的方案进行大规模前瞻性研究。 